\title{
Oximetria Fetal de Pulso: Relação entre a Saturação de Oxigênio do Segundo Período do Parto e o pH da Artéria Umbilical ao Nascimento
}

Fetal Pulse Oximetry: Relationship between Oxygen Saturation at Second Stage of Labor and the Umbilical Artery pH at Birth

Edson Nunes de Morais

\section{RESUM0}

Objetivo: estudar a correlação entre a saturação de oxigênio fetal medida pela oximetria de pulso durante o segundo periodo do parto e o pH da artéria umbilical ao nascimento.

Pacientes e Métodos: a saturação de oxigênio fetal $\left(\mathrm{FSpO}{ }_{2}\right)$ foi monitorizada pela oximetria de pulso durante o segundo periodo do trabalho de parto em 64 gestações simples a termo com apresentação de vértice. $O$ sangue dos vasos umbilicais foi obtido imediatamente após o nascimento para subseqüente medida dos gases e do $\mathrm{pH}$ venoso e arterial. Todos os fetos mantiveram a $\mathrm{FSpO}_{2} \geq 30 \%$ durante o primeiro periodo do parto, até o início do segundo periodo.

Resultados: a média da $\mathrm{FSpO}_{2}$ durante o segundo periodo do parto correlacionou-se significantemente com o $\mathrm{pH}$ da artéria umbilical ao nascimento ( $n=64, r=0,79, p<0,001)$. Não houve correlação significante entre a $\mathrm{FSpO}_{2}$ no segundo periodo do parto e a saturação de oxigênio do sangue da artéria umbilical ao nascimento.

Conclusão: a saturação de oxigênio fetal medida pela oximetria de pulso durante o segundo periodo do trabalho de parto tem uma boa correlação com o $\mathrm{pH}$ da artéria umbilical ao nascimento.

PALAVRAS-CHAVE: Oximetria fetal de pulso. Monitorização fetal. Anóxia perinatal. Trabalho de parto.

\section{Introdução}

A pulso-oximetria é uma técnica nãoinvasiva largamente utilizada por anestesistas, neonatologistas e em unidades de tratamento intensivo para a monitorização contínua da saturação de oxigênio $\left(\mathrm{SpO}_{2}\right)$. A utilização dessa tecnologia para a avaliação e identificação do feto de risco durante o trabalho de parto foi uma conseqüência lógica. A monitorização da saturação de oxigênio fetal $\left(\mathrm{FSpO}_{2}\right)$ durante o parto é uma tecnologia emergente e com potencial para a aplicação clínica ${ }^{5,6}$. Nos últimos 9 anos têm sido

Correspondência:

Edson Nunes de Morais,

Av. Borges de Medeiros, 1699/604

97015-090 - Santa Maria - RS

Tel (55) 222-1942 - Fax (55) 220-8018

e-mail: edson@ccs.ufsm.br envidados esforços para adaptar esse método à monitorização fetal durante a gestação e parto ${ }^{4,11}$.

Os valores absolutos da $\mathrm{FSpO}_{2}$ considerados normais diferem entre os vários autores, bem como variam numa faixa relativamente grande. Johnson et al. ${ }^{8}$ estudaram a $\mathrm{SpO}_{2}$ fetal nos vários estágios do parto e encontraram níveis de $58 \%$ no final do parto, inferiores àqueles do início do trabalho de parto (68\%). Dildy et al. ${ }^{2}$ em um estudo preliminar de 73 casos, descrevem uma média de 57,9 $\pm 10.0 \%$ durante todo o parto. Alguns autores tentaram correlacionar as medidas da $\mathrm{FSpO}_{2}$ durante os últimos minutos do parto com os gases do sangue do cordão. McNamara et al. ${ }^{14}$ relatam uma correlação significante entre a $\mathrm{FSpO}_{2}$ medida 10 minutos antes do nascimento e o $\mathrm{pH}$ da artéria umbilical.

O presente estudo foi realizado com o objetivo de buscar uma possivel correlação entre os valores da $\mathrm{FSpO}_{2}$, medidos por meio da oximetria fetal de 
pulso no segundo período do parto, e o $\mathrm{pH}$ do sangue da artéria umbilical ao nascimento.

\section{Pacientes e Métodos}

A $\mathrm{FSpO}_{2}$ foi medida pela oximetria de pulso durante o segundo período do parto, em 64 parturientes portadoras de gestações únicas de termo, com bolsa rota espontânea ou artificialmente há menos de 12 horas e com apresentação de vértice. Todas as pacientes arroladas no estudo assinaram o termo de consentimento esclarecido e tiveram seus partos no Centro Obstétrico do Hospital Universitário de Santa Maria. O projeto de pesquisa foi previamente aprovado pelas Comissões de Ética e de Pesquisa do Centro de Ciências da Saúde da Universidade Federal de Santa Maria. Os critérios de exclusão foram gestações múltiplas, idade gestacional inferior a 37 semanas, placenta prévia, corioamnionite, sangramento vaginal de origem desconhecida, anomalias uterinas, doenças sexualmente transmissiveis e peso fetal estimado $<2.500$ g. Todos os fetos mostraram, durante o primeiro periodo do trabalho de parto até o início do segundo período, níveis de $\mathrm{FSpO}_{2} \geq 30 \%$.

Para fins de análise dos registros oximétricos considerou-se uma leitura dos 30 minutos prévios ao nascimento, no máximo. Os dados obtidos de cada caso foram examinados pela observação do sinal de oximetria e do tempo anotado entre o desprendimento do tronco fetal e o segmento do registro com sinal aceitável mais recente (até 30 minutos antes do nascimento). Para cada registro, o somatório dos pontos era dividido pelo número total de pontos, consignando-se desta forma a média da $\mathrm{FSpO}_{2}$ para o caso em estudo. Foi considerada como anormal uma $\mathrm{FSpO}_{2}<30 \%$ por mais de 10 minutos entre as contrações. Os valores médios de $\mathrm{FSpO}_{2}$ foram comparados entre faixas de $\mathrm{pH}$ da artéria umbilical divididas em: 7,057,09; 7,10-7,14; 7,15-7,19; 7,20-7,24; e >7,24 além de correlacionados com o pH da artéria umbilical.

As técnicas utilizadas para a cardiotocografia, oximetria fetal de pulso e obtenção do sangue dos vasos umbilicais foram as descritas por Morais et al. ${ }^{15}$ em trabalho anterior. Um sensor de oxigênio fetal descartável da Nellcor Puritan Bennett Inc., Pleasanton, CA, modelo FS-14B, e um cardiotocógrafo da Corometrics Medical Systems Inc., modelo $129 \mathrm{~F}$ foram utilizados para a monitorização fetal durante o trabalho de parto. As amostras de sangue dos vasos umbilicais foram analisadas por meio de equipamentos AVL, Automatic Blood Gas System, modelos 990 e Compact 1, do Laboratório Central de Análises
Clínicas do Hospital Universitário. O peso neonatal, o índice de Apgar e as complicações do recémnascido foram registradas por berçarista do hospital. Todos os recém-nacidos foram seguidos do nascimento até a alta hospitalar.

O programa SPSSPC foi utilizado para a análise estatística dos resultados. O coeficiente de correlação de Pearson foi utilizado para correlacionar as médias de saturação de oxigênio fetal com o $\mathrm{pH}$ e gases do sangue da artéria umbilical e o teste $t$ de Student para comparação das médias de $\mathrm{FSpO}_{2}$ nas várias faixas de $\mathrm{pH}$ do sangue da artéria umbilical.

\section{Resultados}

O tempo total de monitorização foi de 9.472 minutos, com uma média de 148,2 $\pm 110,3$ minutos para cada caso. A média de idade das parturientes foi de 24,2 $\pm 6,8$ anos. Em mais de $50 \%$ dos casos a idade gestacional era igual ou maior que 40 semanas. A média da idade gestacional foi de $39,1 \pm 1,6$ semanas. A maior parte das pacientes estudadas era de nuliparas $(43,8 \%)$ e primiparas $(23,4 \%)$. A paridade variou entre 1 e 9 nascimentos. A grande maioria dos recém-nascidos $(59,4 \%)$ apresentou peso entre 3.000 e 3.999 gramas. A média de peso dos recém-nascidos foi de 3.275 com limites entre 2.510 e 4.490 gramas.

O parto vaginal espontâneo ocorreu em 41 casos $(64,1 \%)$ e o fórcipe foi utilizado em 14 parturientes $(21,8 \%)$. A cesárea foi indicada em 9 oportunidades $(14,1 \%)$, sendo que a desproporção céfalo-pélvica foi a indicação em 4 oportunidades e o período expulsivo prolongado, em três. Dos 64 casos estudados em 10 a $^{\mathrm{SpO}_{2}}$ fetal do $2^{\circ}$ período mostrou níveis inferiores a $30 \%$. No entanto, apenas em 4 oportunidades estes valores estiveram além de 10 minutos consecutivos, o que indicou interrupção da gestação por sofrimento fetal. Todos estes fetos apresentaram $\mathrm{pH}$ da artéria umbilical $<7,15$ ao nascimento ( 2 cesáreas e 2 fórcipes).

Cinco recém-nascidos apresentaram índice de Apgar inferior a 7 no primeiro minuto, sendo que dois permaneceram deprimidos no quinto minuto. No décimo minuto todos haviam recuperado os índices e na alta hospitalar não apresentavam problemas neonatais importantes.

A porcentagem média da $\mathrm{FSpO}_{2}$ no segundo período do trabalho de parto correlacionou-se significantemente com o $\mathrm{pH}$ do sangue da artéria umbilical (Figura 1) ao nascimento $(n=64, r=0,79$, $\mathrm{P}<0,0001)$. Não houve correlação significante entre 
a $\mathrm{SpO}_{2}$ fetal no segundo período do parto e a saturação de oxigênio do sangue da artéria umbilical.

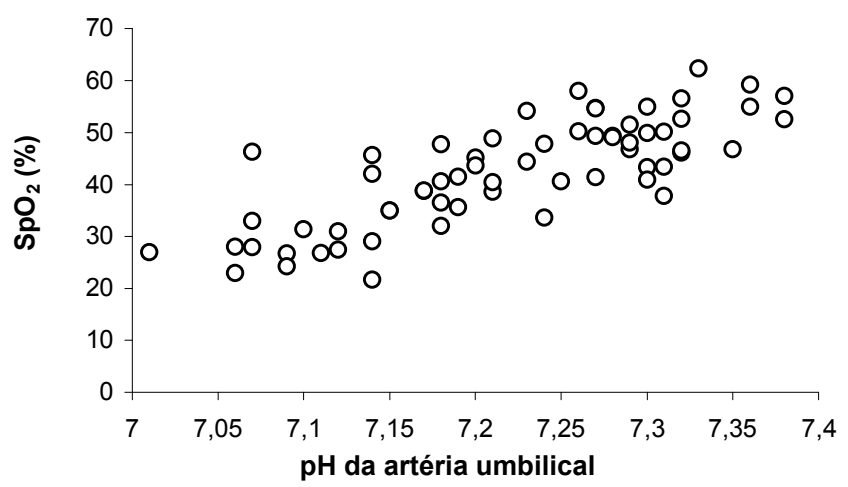

Figura 1 - Correlação entre a $\mathrm{SpO}_{2}$ fetal do segundo período do parto e o pH do sangue da artéria umbilical ao nascimento $(n=64 ; r=0,79 ; p<0,0001)$

$\mathrm{Na}$ Figura 2 encontram-se as médias de $\mathrm{SpO}_{2}$ fetal em função da divisão do $\mathrm{pH}$ da artéria umbilical nas várias faixas estudadas. Não houve diferença significante da $\mathrm{FSpO}_{2}$ nas faixas de $\mathrm{pH}$ compreendidas entre 7,05-7,09 e 7,10-7,14; 7,107,14 e $7,15-7,19 ; 7,15-7,19$ e $7,20-7,24$; e $7,20-$ 7,24 e $>7,24$. Foi observada diferença significante entre as faixas 7,05-7,09 e 7,15-7,19 (p <0,001); 7,05-7,09 e 7,20-7,24 (p <0,002); 7,05-7,09 e $>7,24$ (p <0,0001); 7,10-7,14 e 7,20-7,24 (p $<0,001) ; 7,10-7,14$ e > 7,24 (p <0,005); e 7,15-7,19 e $>7,24(p<0,001)$. É de se notar uma diminuição gradual da $\mathrm{FSpO}_{2}$ à medida que decresce também o pH da artéria umbilical. Dos 16 casos com pH $<7,15$, em 10 a $\mathrm{FSpO}_{2}$ média consignada esteve abaixo de $30 \%$, sendo que em 4 casos a $\mathrm{FSpO}_{2}$ foi $<30 \%$ além de 10 minutos entre as contrações. Quando o pH esteve acima de 7,14, nenhum feto mostrou niveis de $\mathrm{SpO}_{2}$ inferiores a 30\%.

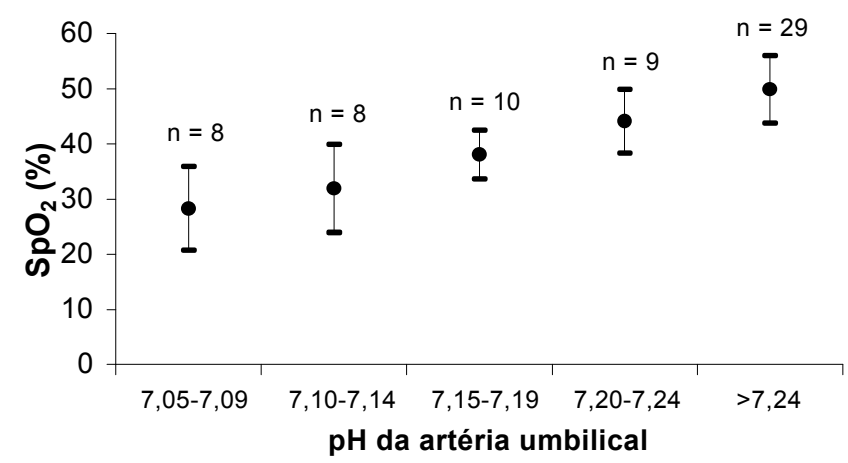

Figura 2 - Médias e desvios-padrão da $\mathrm{SpO}_{2}$ fetal nas várias faixas de $\mathrm{pH}$ da artéria umbilical estudadas.

\section{Discussão}

A oximetria fetal de pulso vem sendo investigada em vários centros internacionais de pesquisa, tendo sido pela primeira vez descrita por Peat et al. ${ }^{16}$ e Johnson \& Lilford ${ }^{9}$. Atualmente vários estudiosos vêm desenvolvendo trabalhos no sentido de estabelecer os padrões de normalidade da $\mathrm{SpO}_{2}$ fetal para o trabalho de parto, além de correlacionálos com outros parâmetros de vitalidade conceptual. Estudos multicêntricos estão em andamento nos Estados Unidos e países da Europa ${ }^{3,7,10}$. O principal objetivo centra-se na busca da padronização do novo método, valores de saturação de oxigênio fetal no primeiro e segundo períodos do parto e a melhoria da especificidade dos parâmetros de cardiotocografia, quando associados aos valores de $\mathrm{FSpO}_{2}$, no prognóstico de recém-nascidos acidóticos. Como conseqüência, busca-se a minimização das taxas de intervenções desnecessárias por hipóxia fetal, quando a oxicardiotocografia for utilizada para avaliar o feto.

O presente estudo demonstrou uma boa correlação entre os valores de $\mathrm{SpO}_{2}$ fetal, consignados no segundo período do parto, e o $\mathrm{pH}$ do sangue da artéria umbilical ao nascimento. Observou-se ainda uma diminuição destes valores à medida que o $\mathrm{pH}$ da artéria umbilical apresentavase mais baixo. No entanto é de se notar que, em vários casos, as condições de normalidade da oxigenação fetal foram observadas mesmo quando o $\mathrm{pH}$ arterial fetal encontrava-se em niveis inferiores a 7,15 . Já numa faixa superior de $\mathrm{pH}(>7,14)$, nenhum feto apresentou niveis de $\mathrm{SpO}_{2}<30 \%$. Butterwegge $^{1}$ observou niveis de $\mathrm{FSpO}_{2}$ entre $25 \%$ e $60 \%$ no segundo período do parto e com pH do sangue da artéria umbilical $>7,20$.

McNamara et al. ${ }^{14}$ estudaram as medidas da $\mathrm{FSpO}_{2}$ durante os últimos 10 minutos do segundo periodo do trabalho de parto. Em 28 casos estudados estes autores encontraram um correlação significante entre a $\mathrm{SpO}_{2}$ fetal e o $\mathrm{pH}$ do sangue da artéria umbilical ( $\mathrm{n}=28, \mathrm{r}=0,63, \mathrm{p}<0,001$ ). Não observaram, por outro lado, correlação estatisticamente significante entre a $\mathrm{FSpO}_{2}$ e a saturação de oxigênio do sangue arterial do cordão umbilical. Luttkus et al. ${ }^{12,13}$ compararam 51 medidas de saturação de oxigênio do sangue capilar do couro cabeludo fetal com leituras de $\mathrm{FSpO}_{2}$. Um coeficiente de correlação de 0,67 foi obtido com valores entre $15 \%$ e $72 \%$ de saturação.

No presente estudo um coeficiente de correlação de 0,79 foi encontrado entre a $\mathrm{FSpO}_{2} \mathrm{e}$ o pH do sangue arterial fetal. Esta correlação não existiu quando foram estudados os niveis de saturação de oxigênio medidos pela oximetria de pulso e aqueles obtidos do sangue de artéria umbilical, semelhantemente aos resultados obtidos por McNamara et al. ${ }^{14}$.

Butterwegge, em $1997^{1}$, estudando 200 casos 
monitorizados pela oximetria fetal de pulso na Alemanha, mostrou que a hipóxia fetal, dada por valores de $\mathrm{pH}$ da artéria umbilical $<7,10$, ocorreu raramente. Observou 14 casos nestas circunstâncias. Já numa faixa de $\mathrm{pH}$ da artéria umbilical entre 7,10 e 7,20 encontrou 32 casos. O autor não estabelece dados relacionados a um $\mathrm{pH}$ arterial de 7,15 (< ou $>$ ), considerado por muitos como limite inferior da normalidade. Se considerarmos niveis de $\mathrm{pH}$ da artéria umbilical $<7,10$, conforme o estabelecido por aquele autor, como limite para determinar a hipóxia conceptual, os resultados do presente estudo demonstram que $6(75 \%)$ de 8 fetos mostraram niveis de $\mathrm{SpO}_{2}<30 \%$. Se arrolarmos os 16 casos com um $\mathrm{pH}$ $<7,15$ (hipóxia fetal), em 10 deles $\left(55,5 \%\right.$ ) a $\mathrm{SpO}_{2}$ fetal apresentou niveis $<30 \%$. No entanto é de se registrar que nem todos os fetos com saturação de oxigênio inferior a 30\%, em algum momento, tiveram diagnóstico de hipóxia pela oximetria de pulso $\left(\mathrm{SpO}_{2}<30 \%\right.$ por $>10$ minutos). Esta é uma análise apenas especulativa, pois o pequeno número de casos de fetos acidóticos de nosso material não nos permite conclusões definitivas. Estudos posteriores mais consistentes envolvendo um maior número de casos são desejáveis, incluindo a perspectiva do estabelecimento do ponto de corte para os valores de $\mathrm{SpO}_{2}$ fetal no segundo periodo do trabalho de parto nas várias faixas de $\mathrm{pH}$ do sangue da artéria umbilical ao nascimento.

Concluindo, a saturação de oxigênio fetal medida pela oximetria de pulso durante o segundo período do trabalho de parto mostrou uma correlação significante com o $\mathrm{pH}$ do sangue da artéria umbilical, em casos em que a $\mathrm{SpO}_{2}$ conceptual se manteve em niveis $\geq 30 \%$ durante o primeiro periodo do parto.

\section{SUMMARY}

Purpose: to study the correlation between fetal oxygen saturation measured by pulse oximetry during second stage of labor and umbilical artery $\mathrm{pH}$ at birth.

Patients and Methods: fetal oxygen saturation ( $\mathrm{FSpO}$ ) was monitored by pulse oximetry during the second stage of labor in 64 singleton pregnancies at term, with vertex presentation. Umbilical blood was sampled immediately after delivery for subsequent measurement of venous and arterial blood gases and $\mathrm{pH}$. All fetuses maintained $\mathrm{FSpO} \mathrm{O}_{2} \geq 30 \%$ through the first stage of labor, until the start of second stage.

Results: the mean $\mathrm{FSpO}_{2}$ at the second stage of labor correlated significantly with umbilical artery $\mathrm{pH}$ at birth $(n=64, r=0.79, p<0.001)$. There was no significant correlation between $\mathrm{FSpO}_{2}$ at the second stage of labor and umbilical artery oxygen saturation at birth.

Conclusion: fetal oxygen saturation measured by pulse oximetry during second stage of labor has a good correlation with umbilical artery $\mathrm{pH}$ at birth.

KEY WORDS: Fetal pulse oximetry. Fetal distress. fetal monitoring.

\section{Referências}

1. Butterwegge M. Fetal pulse oximetry and non-reassuring heart rate. Eur J Obstet Gynecol Reprod Biol 1997;72 (Suppl.1): S63-S66.

2. Dildy GA Clark SL, Loucks CA. Preliminary experience with intrapartum fetal pulse oximetry in humans. Obstet Gynecol 1993;81:630-5.

3. Dildy GA, Clark SL, Garite TJ, Porter TF, Swedlow DB, Varner MW. Current status of the multicenter randomized clinical trial on fetal oxygen saturation monitoring in the United States. Eur J Obstet Gynecol Reprod Biol 1997;72 (Suppl.1): S43-S50.

4. Dildy GA, Clark SL, Loucks CA. Intrapartum fetal pulse oximetry: Past, present and future. Am J Obstet Gynecol 1996;175:1-9.

5. Edrich T, Rall G, Knitza R. Fetal pulse oximetry: Influence of tissue blood content and hemoglobin concentration in a new in-vitro model. Eur J Obstet Gynecol Reprod Biol 1997;72 (Suppl.1): S29-S34.

6. Elchalal U, Weissman A, Abramov Y, Abramov D, Weinstein D. Intrapartum fetal pulse oximetry: Present and future. Gynecol Obstet 1995;50:131-137.

7. Goffinet F, Langer B, Carbonne B, Berkane N, Tardif D, Le Goueff $\mathrm{F}$ et al. Multicenter study on the clinical value of fetal pulse oximetry. I. Methodologic evaluation. Am J Obstet Gynecol 1997;177:1238-46.

8. Johnson N, Johnson VA, Fisher J, Jobbings B, Bannister J, Lilford RJ. Fetal monitoring with pulse oximetry. Br J Obstet Gynaecol 1991;98:36-41.

9. Johnson $\mathrm{N}$, Lilford RJ. Continuous intrapartum measurement of fetal oxygen saturation (Letter). Lancet 1988;2:517.

10. Kuhnert M, Seelbach-Goebel B, Butterwegge M. Predictive agreement between the fetal arterial oxygen saturation and fetal scalp $\mathrm{pH}$ : Results of the German multicenter study. Am J Obstet Gynecol 1998;178:330-5.

11. Lewinsky RM, Fine I. Antepartum fetal pulse oximetry. Eur J Obstet Gynecol Reprod Biol 1997;72 (Suppl.1): S81-S85.

12. Luttkus A, Fengler TW, Friedmann W, Dudenhausen JW. Continuous monitoring of fetal oxygen saturation by pulse oximetry. Obstet Gynecol 1995;85:183-6.

13. Luttkus A, Fengler TW, Friedmann W, Nimpsch R, Dudenhausen JW. Fetal oxygen saturation in cases with suspected hypoxia and normal cardiotocogram: A prospective investigation about fetal pulse oximetry. Z Geburtshilfe Perinatol 1994;198:62-6.

14. McNamara H, Chung DC, Lilford R, Johnson N. Do fetal pulse oximetry readings at delivery correlate with blood oxygenation and acidemia? $\mathrm{Br} \mathrm{J}$ Obstet Gynaecol 1992;99:735-8.

15. Morais E, Gallarreta FPM, Spara P. Saturação de oxigênio fetal medida pela oximetria fetal de pulso durante o trabalho de parto: Relações com o pH de artéria umbilical. Rev Bras Ginecol Obstet 1999;21:141-6.

16. Peat S, Booker M, Lanigan C, Ponte J. Continuous intrapartum measurement of fetal oxygen saturation (Letter). Lancet 1988;2:213. 